\title{
A Pragmatic Non-Blocking Concurrent Directed Acyclic Graph
}

\author{
Sathya Peri ${ }^{1}$, Muktikanta $\mathrm{Sa}^{2}$, Nandini Singhal ${ }^{3}$ \\ Department of Computer Science \& Engineering \\ Indian Institute of Technology Hyderabad, India \\ $\left\{{ }^{1}\right.$ sathya_p, ${ }^{2}$ cs15resch11012\}@iith.ac.in, ${ }^{3}$ nandini12396@gmail.com
}

\begin{abstract}
In this paper, we have developed two algorithms for maintaining acyclicity in a concurrent directed graph. The first algorithm is based on a wait-free reachability query and the second one is based on partial snapshot-based obstruction-free reachability query. Interestingly, we are able to achieve the acyclic property in the dynamic setting without the need of helping using descriptors by other threads or clean double collect mechanism. We present a proof to show that the graph remains acyclic at all times in the concurrent setting. We also prove that the acyclic graph data-structure operations are linearizable. We implement both the algorithms in $\mathrm{C}++$ and test through a number of micro-benchmarks. Our experimental results show an average of $7 \mathrm{x}$ improvement over the sequential and global lock implementation.
\end{abstract}

keywords: acyclic graph concurrent data structure linearizability lock-freedom.

\section{Introduction}

A graph is a common data-structure that can model many real-world objects and pairwise relationships among them. Graphs have a huge number of applications in various field like social networking, VLSI design, road network, graphics, blockchains and many more. Usually, these graphs are dynamic in nature, that is, they undergo dynamic changes like addition and removal of vertices and/or edges [7]. These applications also need data-structure which supports dynamic changes and can expand at run-time depending on the availability of memory in the machine.

Nowadays, multi-core systems have become ubiquitous. To fully harness the computational power of these systems, it has become necessary to design efficient data-structures which can be executed by multiple threads concurrently. In the past decade, there have been several efforts to port sequential data-structures to a concurrent setting, like stacks, queues, sets, trees.

Most of these data-structure use locks to handle mutual exclusion while doing any concurrent modifications. However, in an asynchronous shared-memory system, where an arbitrary delay or a crash failure of a thread is possible, a lock-based implementation is vulnerable to arbitrary delays or deadlock. For instance, a thread could acquire a lock and then sleep (or swapped out) for a long time. Or the thread could be involved cyclic wait with other threads while obtaining locks or crash after obtaining the lock.

On the other hand, in a lock-free data-structure, threads do not acquire locks. Instead, they use atomic hardware instructions such as compare-and-swap, test-and-set etc. These instructions ensure that at least one non-faulty thread is guaranteed to finish its operation in a finite number of steps. Therefore, lock-free data-structures are highly scalable and naturally fault-tolerant.

Although several concurrent data-structures have been developed, concurrent graph data-structures and the related operations are still largely unexplored. In several graph applications, one of the crucial requirements is preserving acyclicity. Acyclic graphs are often applied to problems related 
to databases, data processing, scheduling, finding the best route in navigation, data compression, blockchains etc. Applications relying on graphs mostly use a sequential implementation and the access to the shared data-structures are synchronized through the global locks, which causes serious performance bottlenecks.

A relevant application is Serialization Graph Testing (SGT) in Databases [14, Chap 4] and Transactional Memory (TM) [13]. SGT requires maintaining an acyclic graph on all concurrently executing (database or TM) transactions with edges between the nodes representing conflicts among them. In a concurrent scenario, where multiple threads perform different operations, maintaining acyclicity without using locks is not a trivial task. Indeed, it requires every shared memory access to be checked for the violation of the acylic property, which necessitates optimization of the steps in all these operations.

Apart from SGT, maintaining acyclic graphs can be very useful in blockchains. Several popular blockchains maintain acyclic graphs such as tree structure (Bitcoin [2], Ethereum [3] etc.) or general DAGs (Tangle [12])).

\subsection{Contributions}

In this paper, we present an efficient non-blocking concurrent acyclic directed graph data-structure and its operations are similar to Chatterjee et. al. [4] with some elegant modifications. The contributions of our work are summarized below:

1. We describe an Abstract Data Type (ADT) that maintains an acyclic directed graph $G=$ $(V, E)$. It comprises of the following operations on the sets $V$ and $E$ : (1) Add Vertex: AcyAddV (2) Remove Vertex: AcyRemV, (3) Contains Vertex: AcyConV (4) Add Edge: AcyAddE (5) Remove Edge: AcrRemE and (6) Contains Edge: AcyConE. The ADT remains acyclic after completion of any of the above operations in $G$. The acyclic graph is represented as an adjacency list like in [4].

2. We present an efficient concurrent non-blocking implementation of the ADT (Section 3). We present two approaches for maintaining acyclicity: the first one is based on a wait-free reachability query and the second one is based on obstruction-free reachability query similar to the GetPath operation of Chatterjee et al. [4] (Section 4).

3. We prove the correctness by showing the operations of the concurrent acyclic graph datastructure are linearizable [10]. We also prove the non-blocking progress guarantee: (a) The operations ACyCONV and ACYCONE are wait-free, only if the vertex keys are finite; (b) Among the two algorithms for maintaining acyclicity, we show that the first algorithm based on searchability is wait-free, whereas the second algorithm based on reachability queries is obstruction-free and (c) The operations AcyAddV, AcrRemV, AcyConV, AcyAddE, AcrRemE, and AcyConE are lock-free. Section 5.

4. We evaluated the non-blocking algorithms in $\mathrm{C}++$ implementation and tested through a number of micro-benchmarks. Our experimental results depict on an average of $7 \mathrm{x}$ improvement over the sequential and global lock implementation (Section 6).

\subsection{Related Work}

Kallimanis and Kanellou [11] presented a concurrent graph that supports wait-free edge updates and traversals. They represent the graph using adjacency matrix, with bounded number of vertices. As a result, their graph data-structure does not allow any insertion or deletion of vertices after 
initialization of the graph. This may not be adequate for many real-world applications which need dynamic modifications of vertices as well as unbounded graph size.

A recent work by Chatterjee et al. [4] proposed a non-blocking concurrent graph data-structure which allowed multiple threads to perform dynamic insertion and deletion of vertices/edges. Our paper extends this data-structure to maintain acyclicity of a directed graph. Their algorithm does not allow the the graph to maintain acyclicity after any update operation.

\subsection{Overview of the Algorithm Design}

Before getting into the technical details (in Section 3) of the algorithm, we first provide an overview of the design. We implement an acyclic concurrent unbounded directed graph based on both existing lock-free linked-list [8] and the concurrent graph data-structure [4]. The vertex-nodes are placed in a sorted linked-list and the neighboring vertices of each vertex-node are placed in a rooted sorted linked-list of edge-nodes. To achieve efficient graph traversal, we maintain a pointer from each edgenode to its corresponding vertex-node. Each vertex-node's edge-list and vertex-list are lock-free with concurrent updates and lookup operations.

As we know that lock-freedom is not composable [6] and our algorithm is a composition of lock-free operations, we prove the liveness of our algorithm independent of lock-free list arguments. In addition to that, we also propose some graceful optimizations for the concurrent acyclic graph operations that not only enhance the performance but also simplify the design.

Our main requirement is preserving acyclicity and one can see that a cycle is created only after inserting an edge to the graph. So, after inserting a new edge to the graph we verify if the resulting graph is acyclic or not. If it creates a cycle, we simply delete that edge from the graph. However, the challenge is that these intermediate steps must be oblivious to the user and the graph must always appear to be acyclic. We ensure this by adding a transit field to the edges that are temporarily added.

To verify the acyclic property of the graph we propose two efficient algorithms: first one based on a wait-free reachability query and the second one based on obstruction-free reachability query similar to the GETPATH operation of [4]. Both the reachability algorithms perform the breadth-first search (BFS) traversal. For the sake of efficiency, we implement BFS traversal in a non-recursive manner. However, in order to achieve the overall performance we do not help the reachability queries.

\section{System Model and Preliminaries}

The Memory Model. We consider an asynchronous shared-memory model with a finite set of $p$ processors accessed by a finite set of $n$ threads. The non-faulty threads communicate with each other by invoking methods on the shared objects. We execute our acyclic graph data-structure on a shared-memory multi-core with multi-threading enabled which supports atomic read, write, fetch-and-add (FAA) and compare-and-swap (CAS) instructions.

A FAA $(x, a)$ instruction atomically increments the value at the memory location $x$ by the value a. Similarly, a $\operatorname{CAS}\left(x, a, a^{\prime}\right)$ is an atomic instruction that checks if the current value at a memory location $x$ is equivalent to the given value $a$, and only if true, changes the value of $x$ to the new value $a^{\prime}$ and returns true; otherwise the memory location remains unchanged and the instruction returns false. Such a system can be perfectly realized by a Non-Uniform Memory Access (NUMA) computer with one or more multi-processor CPUs.

Correctness. We consider linearizability proposed by Herlihy \& Wing [10] as the correctness criterion for the graph operations. We assume that the execution generated by a data-structure is 
a collection of operation invocation and response events. Each invocation of a method call has a subsequent response. An execution is linearizable if it is possible to assign an atomic event as a linearization point $(L P)$ inside the execution interval of each operation such that the result of each of these operations is the same as it would be in a sequential execution in which the operations are ordered by their LPs [10].

Progress. The progress properties specifies when a thread invoking operations on the shared memory objects completes in presence of other concurrent threads. In this context, we provide an acyclic graph implementation with operations that satisfies lock-freedom, based on the definitions in Herlihy \& Shavit [9].

\section{The Data Structure}

\subsection{Abstract Data Type}

An acyclic graph is defined as a directed graph $G=(V, E)$, where $V$ is the set of vertices and $E$ is the set of directed edges. Each edge in $E$ is an ordered pair of vertices belonging to $V$. A vertex $v \in V$ has an immutable unique key $k$ denoted by $v(k)$. A directed edge from the vertex $v\left(k_{1}\right)$ to $v\left(k_{2}\right)$ is denoted as $e\left(v\left(k_{1}\right), v\left(k_{2}\right)\right) \in E$. For simplicity, we denote $e\left(v\left(k_{1}\right), v\left(k_{2}\right)\right)$ as $e\left(k_{2}\right)$, which means the $v\left(k_{1}\right)$ has a neighbouring vertex $v\left(k_{2}\right)$. For a concurrent acyclic graph, we define following ADT operations:

1. The $\operatorname{Acy} \operatorname{AdDV}(k)$ adds a vertex $v(k)$ to $V$, only if $v(k) \notin V$ and then returns true, otherwise it returns false.

2. The $\operatorname{Acy} \operatorname{Rem} V(k)$ deletes a vertex $v(k)$ from $V$, only if $v(k) \in V$ and then returns true, otherwise it returns false. Once a vertex $v(k)$ is deleted successfully all its outgoing and incoming edges also removed.

3. The $\mathrm{ACyCONV}(k)$ if $v(k) \in V$ it returns true, otherwise it returns false.

4. The $\operatorname{Acy} \operatorname{AdDE}\left(k_{1}, k_{2}\right)$ adds an edge $e\left(v\left(k_{1}\right), v\left(k_{2}\right)\right)$ to $E$, only if $e\left(v\left(k_{1}\right), v\left(k_{2}\right)\right) \notin E$ and $v\left(k_{1}\right) \in V$ and $v\left(k_{2}\right) \in V$ and it does not create any cycle then it returns EDGE ADDED. If $v\left(k_{1}\right) \notin V$ or $v\left(k_{2}\right) \notin V$, it returns VERTEX NOT PRESENT. If $e\left(v\left(k_{1}\right), v\left(k_{2}\right)\right) \in E$, it returns EDGE ALREADY PRESENT.

5. The $\operatorname{AcyRemE}\left(k_{1}, k_{2}\right)$ deletes the edge $e\left(v\left(k_{1}\right), v\left(k_{2}\right)\right)$ from $E$, only if $e\left(v\left(k_{1}\right), v\left(k_{2}\right)\right) \in E$ and $v\left(k_{1}\right) \in V$ and $v\left(k_{2}\right) \in V$ then it returns EDGE REMOVED. If $v\left(k_{1}\right) \notin V$ or $v\left(k_{2}\right) \notin V$, it returns VERTEX NOT PRESENT. If $e\left(v\left(k_{1}\right), v\left(k_{2}\right)\right) \notin E$, it returns EDGE NOT PRESENT.

6. The $\operatorname{AcyConE}\left(k_{1}, k_{2}\right)$ if $e\left(v\left(k_{1}\right), v\left(k_{2}\right)\right) \in E$ and $v\left(k_{1}\right) \in V$ and $v\left(k_{2}\right) \in V$ then it returns EDGE PRESENT, otherwise it returns VERTEX OR EDGE NOT PRESENT.

\subsection{The data-structure}

The proposed concurrent non-blocking acyclic graph data-structure is constructed based on existing lock-free linked-list [8] and the concurrent graph data-structure [4]. The VNode, ENode and BFSNode structures and the adjacency list representation of an acyclic graph are shown in Figure 1. The VNode structure has five fields, two pointers vnext and enext, an immutable key $k$, an atomic counter ecount, and a VisitedArray array. The use of ecount and VisitedArray is described in the later section. The pointer vnext is an atomic pointer pointing to the next vNode in the vertex-list, whereas, an enext pointer points to the edge head of the edge-list of a VNode. Similarly, 
already present earlier or not. If key is not present earlier it returns EDGE NOT PRESENT otherwise it attempts to remove curre in two steps, (a) atomically marks the enext of curre using a CAS (Line 102), and (b) atomically updates the enext of the prede to point to the enext of curre using a CAS (Line 104). On any unsuccessful CAS, it reattempted. After successful CAS it returns EDGE REMOVED.

Similarly, an AcyConE $\left(k_{1}, k_{2}\right)$ operation, in Lines 43 to 57 , validates the presence of the corresponding VNodes. Then it traverses the edge-list of $v\left(k_{1}\right)$ in a wait-free manner skipping all logically marked ENodes until it finds an edge with its key greater than or equal to $k_{2}$. Once it reaches the appropriate ENode, checks its key value equal to $k_{2}$ and it is unmarked and not in TRANSIT state and also $v\left(k_{1}\right)$ and $v\left(k_{2}\right)$ are unmarked, then it returns EDGE PRESENT otherwise it returns VERTEX OR EDGE NOT PRESENT. Like ACYCONV, we also do not allow ACYCONE for any helping thread in the process of traversal.

\subsection{Wait-free Single Collect Reachable Algorithm}

In this section, we describe one of our algorithms to detect the cycle of a concurrent graph in a waitfree manner. As we mentioned earlier that a cycle can be formed only after adding an edge to the graph. The $\operatorname{SCRCBL}\left(k_{1}, k_{2}\right)$ operation, in Lines 111 to 136 , performs non-recursive BFS traversal starting from the vertex $v\left(k_{1}\right)$. A reader can refer [5] to know the working of the BFS traversals in graphs. In the process of BFS traversal, it explores the VNodes which are reachable from $v\left(k_{1}\right)$ and unmarked. If it reaches $v\left(k_{2}\right)$, then it terminates by returning true to the ACYADDE operation. Then ACYADDE deletes $e\left(k_{2}\right)$ by setting enext pointer from the TRANSIT state to MARKED state and returns CYCLE. If it is unable to reach $v\left(k_{2}\right)$ from $v\left(k_{1}\right)$ after exploring all reachable VNodes through TRANSIT or ADDED or unmarked ENodes, then it terminates by returning false to the ACYADDE operation. Then ACYADDE adds $e\left(k_{2}\right)$ by setting enext pointer from the TRANSIT state to ADDED state and then it returns EDGE ADDED, which preserves the acyclic property after ACYADDE.

In the process of BFS traversal, we used a VisitedArray ( size the same as the number of threads) to put all the visited vNodes locally. This is because multiple threads repeatedly invoke reachable operation concurrently, a boolean variable or a boolean array would not suffice like in case of sequential execution. We used a thread local variable cnt as a counter for the number of repeated traversals by a thread. So, a VisitedArray slot keeps counter cnt value (see Line 115).

However, an ENode in TRANSIT state cannot be removed by any other concurrent thread other than the thread that added it, only if it creates a cycle. The threads which are performing cycle detection can see all the ENodes in TRANSIT or ADDED state. Further, a concurrent ACYCONE operation will ignore all the ENodes with TRANSIT state. This ensures that when an ENode is in the ADDED state an ACYADDE operation return EDGE ADDED. Figure 7 depicts an example of how acyclic property is preserved with concurrent ACYADDE and ACYCONE execution and shows one possible LP case.

A side-effect to be observed here is that this may allow false positives. This means that the algorithm may detect a cycle even though the graph does not contain one. This can happen in the following scenario; two threads $T_{1}$ and $T_{2}$ are adding edges lying in the path of a single cycle. In this case, both threads detect that the newly added ENode (in TRANSIT state) has led to the formation of a cycle and both may delete their respective edges. However, in a sequential execution, only one of the edges will be removed. But, we allow this as the resulting graph at the end of each operation is still acyclic. In other words, false positives do not violate our correctness notion, linearizability.

The proof of the acyclicity is given in Lemma 1(see Section A). 

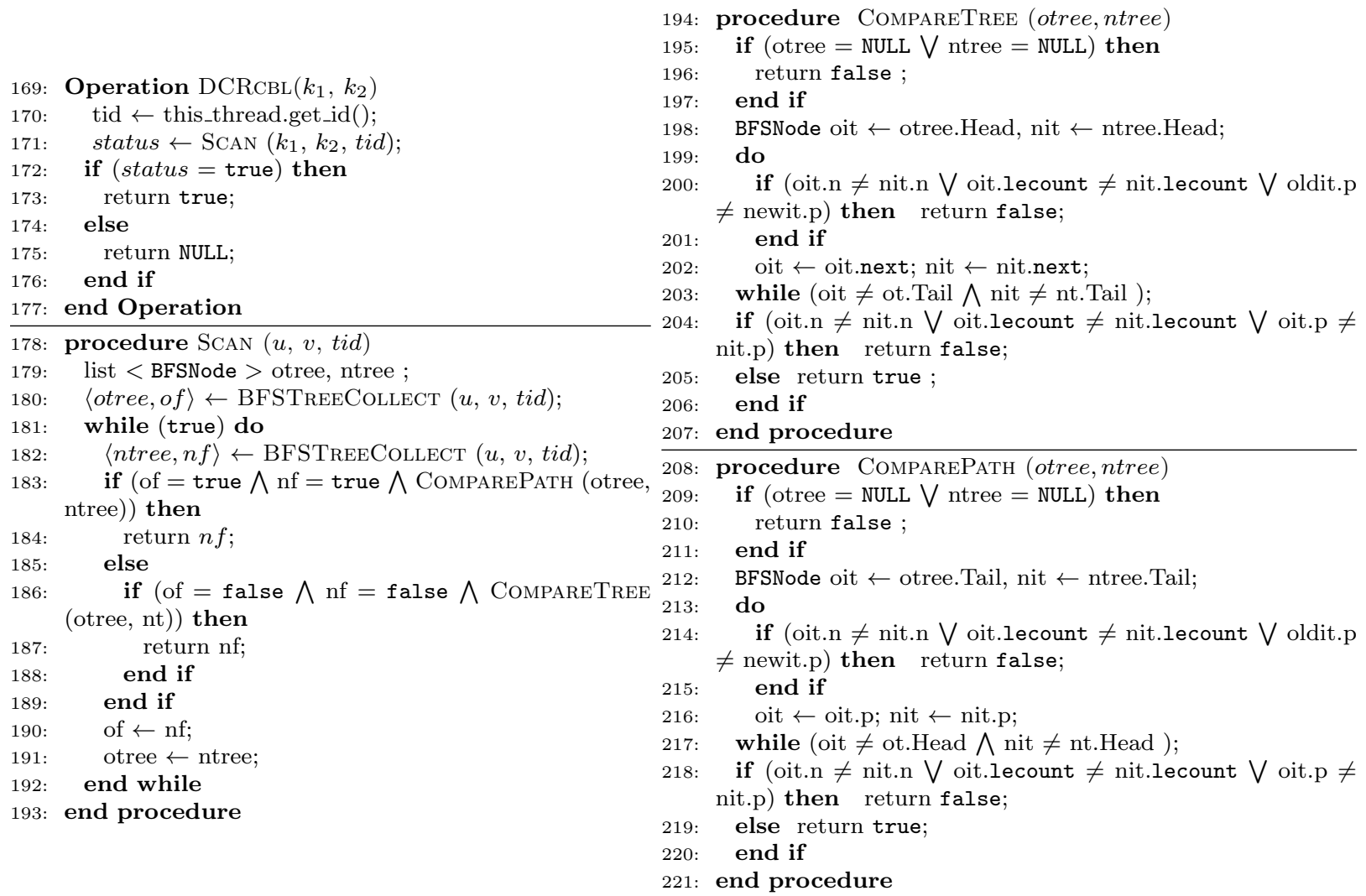

Figure 5: Pseudo-codes of DCRcbl, Scan, CompareTree and ComparePath.

\subsection{Obstruction-free Double Collect Reachable Algorithm}

To overcome the false positives issues in case of SCRCBL algorithm, we proposed an obstructionfree reachability, DCRCBL algorithm, which is designed based on the atomic snapshot algorithm by Afek et al. [1] and reachable algorithm by Chatterjee et al. [4]. The $\operatorname{DCRCBL}\left(k_{1}, k_{2}\right)$ operation, in Lines 169 to 177, performs SCAN starting from $v\left(k_{1}\right)$. It checks whether $v\left(k_{2}\right)$ is reachable from $v\left(k_{1}\right)$. This reachable information is returned to the ACYADDE operation and then ACYADDE decides whether to add $e\left(k_{2}\right)$ ( is in the TRANSIT state) to the edge-list of $v\left(k_{1}\right)$ or not.

The ScAN method, in Lines 178 to 193, first creates two BFS-trees, otree and ntree to hold the VNodes in two consecutive BFS traversal. It performs repeated BFS-tree collection by invoking BFSTreeCollect until two consecutive collects are equal. The BFSTreeCollect procedure, in Lines 137 to 168, performs non-recursive BFS traversal starting from the vertex $v\left(k_{1}\right)$. In the process of BFS traversal, it explores all reachable and unmarked VNodes through adjacency ENodes which are in the TRANSIT or ADDED or unmarked state. However, it keeps adding all these VNodes in the $b \operatorname{Tree}($ see Line $143,154,160)$. If it reaches $v\left(k_{2}\right)$, then it terminates by returning $b T r e e$ and a reachable status true (Line 155) to the SCAN method. If it is unable to reach $v\left(k_{2}\right)$ from $v\left(k_{1}\right)$ after exploring all reachable VNodes, then it terminates by returning bTree and a reachable status false (Line 167) to the SCAN method.

If two consecutive BFSTREECOLLECT method return the same boolean status value true then we invoke COMPAREPATH to compare the two BFS-trees are equal or not. If both trees are equal, then SCAN method returns true to DCRCBL operation, which says $v\left(k_{2}\right)$ is reachable from $v\left(k_{1}\right)$. Then DCRCBL returns true to the ACYADDE operation and then ACYADDE deletes that $e\left(k_{2}\right)$ by setting enext pointer from the TRANSIT state to MARKED state and returns CYCLE, this is because $e\left(k_{2}\right)$ creates a cycle. However, if two consecutive BFSTREECoLLECT methods return 
the same status value false then we invoke COMPARETREe to compare the two BFS-trees are equal or not. If it is, the SCAN method returns false to the DCRCBL operation which says $v\left(k_{2}\right)$ is not reachable from $v\left(k_{1}\right)$. Then DCRCBL returns false to the ACYADDE operation and then ACYADDE adds that $e\left(k_{2}\right)$ by setting enext pointer from the TRANSIT state to ADDED state and then it returns EDGE ADDED. Which confines the acyclic property after ACYADDE. If two consecutive BFSTREECOLLECT method return the same boolean status value true or false but do not match in the ComparePath or CompareTree, then we discard the older BFS-tree and restart the BFSTREECOLLECT.

The ComparePath method, in Lines 208 to 221, compares two BFS-tree based on path and along with the lecount values. It starts from the last BFSNode and follows the parent pointer $p$ until it reaches to the starting BFSNode or any mismatch occurred at any BFSNode. It returns false for any mismatch occurred otherwise returns true. Similarly, the CompareTree method, in Lines 194 to 207, compares two BFS-tree based on all explored VNodes in the process of BFS traversal and along with the lecount values. It starts from the starting BFSNode and follows with the next pointer next until it reaches the last BFSNode or any mismatch occurred at any BFSNode. It returns false for any mismatch occurred and otherwise returns true. We explain the necessary increment of an ecount and requirement of a lecount below.

To capture the modifications along the path of BFS-traversal, we have an atomic counter ecount associated with each vertex. During any edge update operations, before an $e\left(k_{2}\right)$ is physically deleted, the counter ecount of the source vertex $v\left(k_{1}\right)$ is certainly incremented at Line 78 or 103 or 256 either by the operation that logically deleted the $e\left(k_{2}\right)$ or any edge helping operations. To verify the double collect we compare with BFS-tree alone with counter.

\section{Correctness and Progress Guarantee}

In this section, we prove the correctness of our concurrent acyclic graph data-structure based on $L P[10]$ events inside the execution interval of each of the operations.

Theorem 1 The non-blocking concurrent acyclic graph operations are linearizable.

Proof. Based on the return values of the operations we discuss the LPs.

1. $\operatorname{Acy} \operatorname{AddV}(k)$ : Based on the return values we have two cases:

(a) true: The LP be the successful CAS execution at the Line 9.

(b) false: The LP be the atomic read of the vnext pointing to the vertex $v(k)$.

2. $\operatorname{AcrRemV}(k)$ : Based on the return values we have two cases:

(a) true: The LP be the successful CAS execution at the Line 23 (logical removal).

(b) false: If there is a concurrent ACYREmVoperation op, that removed $v(k)$ then the LP be just after the LP of op. If $v(k)$ did not exist in the vertex-list then the LP be at the invocation of ACYREMV.

3. $\mathrm{ACyCONV}(k)$ : Based on the return values we have two cases:

(a) true: The LP be the atomic read of the vnext pointing to the vertex $v(k)$.

(b) false: The LP be the same as returning false ACYREMV, the case $2 b$.

4. $\operatorname{ACY} \operatorname{AdDE}\left(k_{1}, k_{2}\right)$ : Based on the return values we have four cases: 
(a) EDGE ADDED:

i. With no concurrent $\operatorname{AcyRemV}\left(k_{1}\right)$ or $\operatorname{AcyRemV}\left(k_{2}\right)$ : The LP be the successful CAS execution at the Line 75.

ii. With concurrent $\operatorname{AcyRemV}\left(k_{1}\right)$ or $\operatorname{AcrRemV}\left(k_{2}\right)$ : The LP be just before the first remove's LP.

(b) CYCLE:

i. With no concurrent AcyRemV $\left(k_{1}\right)$ or $\operatorname{AcyRemV}\left(k_{2}\right)$ : The LP be the at the Line 81.

ii. With concurrent AcyRemV $\left(k_{1}\right)$ or AcrRemV $\left(k_{2}\right)$ : The LP be just before the first remove's LP.

(c) EDGE ALREADY PRESENT:

i. With no concurrent $\operatorname{AcYREmV}\left(k_{1}\right)$ or $\operatorname{AcyRemV}\left(k_{2}\right)$ : The LP be the atomic read of the enext pointing to the ENode $e\left(k_{2}\right)$ in the edge-list fo the vertex $v\left(k_{1}\right)$.

ii. With concurrent $\operatorname{AcyRemV}\left(k_{1}\right)$ or $\operatorname{AcyRemV}\left(k_{2}\right)$ or $\operatorname{AcyRemE}\left(k_{1}, k_{2}\right)$ : The LP be just before the first remove's LP.

(d) VERTEX NOT PRESENT:

i. At the time of invocation of $\operatorname{ACY} \operatorname{ADDE}\left(k_{1}, k_{2}\right)$ if both vertices $v\left(k_{1}\right)$ and $v\left(k_{2}\right)$ were in the vertex-list and a concurrent ACYREMVremoves $v\left(k_{1}\right)$ or $v\left(k_{2}\right)$ or both then the LP be the just after the LP of the earlier ACYREMV.

ii. At the time of invocation of $\operatorname{ACY} \operatorname{ADdE}\left(k_{1}, k_{2}\right)$ if both vertices $v\left(k_{1}\right)$ and $v\left(k_{2}\right)$ were not present in the vertex-list, then the LP be the invocation point itself.

5. $\operatorname{AcyRemE}\left(k_{1}, k_{2}\right)$ : Based on the return values we have three cases:

(a) EDGE REMOVED:

i. With no concurrent AcyRemV $\left(k_{1}\right)$ or AcyRemV $\left(k_{2}\right)$ : The LP be the successful CAS execution at the Line 102(logical removal).

ii. With concurrent $\operatorname{AcyRemV}\left(k_{1}\right)$ or $\operatorname{Acr} \operatorname{RemV}\left(k_{2}\right)$ : The LP be just before the first remove's LP.

(b) EDGE NOT PRESENT: If there is a concurrent ACYREMEoperation removed $e\left(k_{1}, k_{2}\right)$ then the LP be the just after its LP, otherwise at the invocation of $\operatorname{ACYREME}\left(k_{1}, k_{2}\right)$ itself.

(c) VERTEX NOT PRESENT: The LP be the same as the case ACYADDEreturning "VERTEX NOT PRESENT" $4 \mathrm{~d}$.

6. $\operatorname{Acy} \operatorname{ConE}\left(k_{1}, k_{2}\right)$ : Similar to AcrRemE, based on the return values we have three cases:

(a) EDGE PRESENT:

i. With no concurrent AcrRemV $\left(k_{1}\right)$ or AcyRemV $\left(k_{2}\right)$ : The LP be the atomic read of the enext pointing to the ENode $e\left(k_{2}\right)$ in the edge-list fo the vertex $v\left(k_{1}\right)$.

ii. With concurrent $\operatorname{AcyRemV}\left(k_{1}\right)$ or $\operatorname{AcrRemV}\left(k_{2}\right)$ or $\operatorname{AcrRemE}\left(k_{1}, k_{2}\right)$ : The LP be just before the first remove's LP.

(b) VERTEX NOT PRESENT: The LP be the same as that of the ACYADDE's returning "VERTEX NOT PRESENT" case.

(c) VERTEX OR EDGE NOT PRESENT: The LP be the same as that of the ACYREME's returning "EDGE NOT PRESENT" and ACYADDE's returning "VERTEX NOT PRESENT" cases. 
From the above discussion once can notice that each operation's LPs lies in the interval between the invocation and the return steps. For any invocation of an ACYADDV $(k)$ operation the traversal terminates at the VNode whose key is just less than or equal to $k$. Similar reasoning also true for invocation of an $\operatorname{ACY} \operatorname{ADDE}\left(k_{1}, k_{2}\right)$ operation. Both the operations do the traversal in the sorted vertex-list and edge-list to make sure that a new VNode or ENode does not break the invariant of the acyclic graph data-structure. The AcyRemVand AcyREmEdoes not break the sorted order of the lists. Similarly the non update operations do not modify the data-structure. Thus we concluded that all acyclic graph operations maintain the invariant across the LPs.

This completes the proof of the linearizability.

Theorem 2 The presented concurrent acyclic graph algorithm

(i) The operations AcyConV, AcyConE and SCRCBL are wait-free, if the vertex keys is finite.

(ii) The operation DCRCBL is obstruction-free.

(iii) The operations AcyAddV, AcrRemV, AcyConV, AcyAddE, AcyRemE, and AcyConE are lock-free.

Proof. If the set of keys is finite, then the size of the acyclic graph has a fixed upper bound. This means there are only finite number of vNodes in between sentinel vh and vt in the vertex-list. If any non-faulty thread invokes an $\mathrm{ACYCONV}$, it terminates on reaching vt with finite number of steps. Similar argument can be brought for an ACYConEoperation. Also one can see that SCRCBLoperation never returns true with concurrent update operations and it terminates with finite number of steps. This shows the (i).

Like SCRCBLoperation, a DCRCBL, a COMPARETREE or a COMPAREPATH operations never returns true with concurrent update operations, which impose the While loop Line 181 in SCAN method to not terminate. So, unless a non-faulty thread has taken the steps in isolation a DCRCBLoperation will never returns. This shows the (ii).

Whenever an insertion or a deletion operation is blocked by a concurrent delete operation by the way of a marked pointer, then that blocked operations is helped to make safe return. Generally, insertion and lookup operations do not need help by a concurrent operation. So, if any random concurrent execution consist of any concurrent data-structure operation, then at least one operation finishes its execution in a finite number of steps taken be a non-faulty thread. Therefore, the acyclic graph operations AcyAddV, AcyRemV, AcyConV, AcyAddE, AcyRemE, and AcyConEare lock-free. This shows the (iii).

\section{Experimental Evaluation}

We performed our tests on 56 cores machine with Intel Xeon (R) CPU E5-2630 v4 running at 2.20 $\mathrm{GHz}$ frequency. Each core supports 2 logical threads. Every core's L1 has 64k, L2 has 256k cache memory are private to that core; L3 cache (25MB) is shared across all cores of a processor. The tests were performed in a controlled environment, where we were the sole users of the system. The implementation $^{\mathrm{a}}$ has been done in $\mathrm{C}++$ (without any garbage collection) and threading is achieved by using Posix threads. All the programs were optimized at O3 level.

We start our experiments by setting an initial directed graph with 1000 vertices and nearly $\left(\begin{array}{c}1000 \\ 2\end{array}\right) / 4 \approx 125000$ edges added randomly. Then we create fixed number of threads with each thread randomly performing a set of operations chosen by a particular workload distribution. We evaluate

\footnotetext{
${ }^{\mathrm{a}}$ The source code is available on https://github.com/PDCRL/ConcurrentGraphDS.
} 
(a) High Lookup

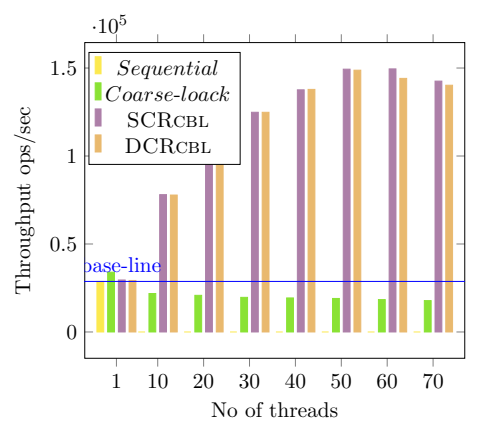

(b) Equal Lookup and Update

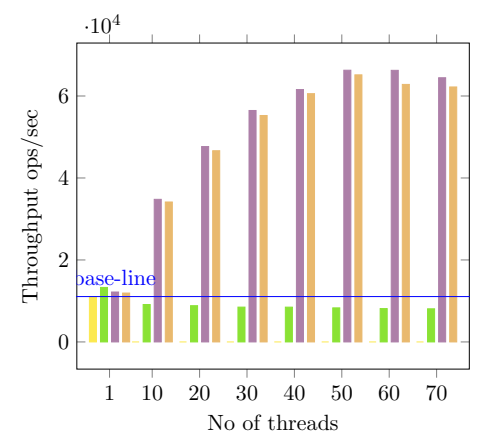

(c) High Update

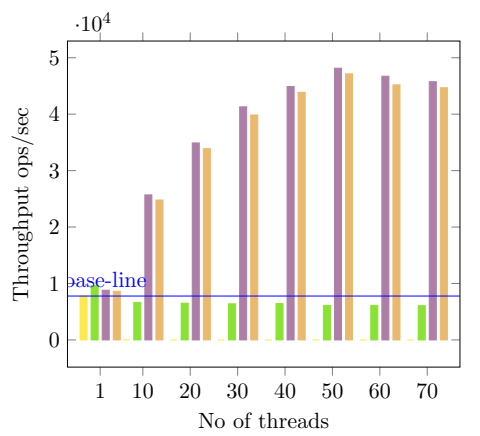

Figure 6: Acyclic Graph Data-Structure.

the number of operations finished their execution in unit time and then calculate the throughput. We run each experiment for 20 seconds and the final data point values are collected after taking an average of 7 iterations. We present the results for the following workload distributions for acyclic directed graph over the ordered set of operations A ACYADDV, ACYREMV, ACYConV, AcyAddE, AcrRemE, AcyConE\} as: (1) High Lookup: $(2.5 \%, 2.5 \%, 45 \%, 2.5 \%, 2.5 \%, 45 \%)$, see the Figure 6a. (2) Equal Lookup and Update: $(12.5 \%, 12.5 \%, 25 \%, 12.5 \%, 12.5 \%, 25 \%$ ), see the Figure 6b. (3) High Update: (22.5\%, 22.5\%, 5\%, 22.5\%, 22.5\%, 5\%), Figure 6c.

From Figure 6, we notice that both SCRCBL and DCRCBL algorithms perform well with the number of threads in comparison with sequential and coarse-lock based version. The wait-free single collect reachable algorithm performs better than obstruction-free double collect reachable algorithm. However, the performance of the coarse-grained lock-based decreases with the number of threads. Moreover also it performs worse than even the sequential implementation. On average, both the non-blocking algorithms perform nearly $7 \times$ times higher throughput over the sequential implementation.

\section{Conclusion}

In this paper, we presented two efficient non-blocking concurrent algorithms for maintaining acyclicity in a directed graph where vertices and edges are dynamically inserted and/or deleted. The first algorithm is based on a wait-free reachability query and the second one is based on partial snapshotbased obstruction-free reachability query. Both these algorithms maintain the acyclic property of the graph throughout the concurrent execution. We prove that the acyclic graph data-structure operations are linearizable. We also present a proof to show that the graph remains acyclic at all times in the concurrent setting. We evaluated both the algorithms in $\mathrm{C}++$ implementation and tested through a number of micro-benchmarks. Our experimental results performed on an average of $7 \mathrm{x}$ improvement over the sequential implementation while the coarse lock implementation performs worse than the sequential one.

\section{References}

[1] Yehuda Afek, Hagit Attiya, Danny Dolev, Eli Gafni, Michael Merritt, and Nir Shavit. Atomic Snapshots of Shared Memory. J. ACM, 40(4):873-890, 1993.

[2] Jerry Brito and Andrea O'Sullivan. Bitcoin: A primer for policymakers. mercatus center at george mason university,, 2013. 
[3] V Buterin. Ethereum: a next generation smart contract and decentralized application platform, https://github.com/ethereum/wiki/wiki/white-paper, 2013.

[4] Bapi Chatterjee, Sathya Peri, Muktikanta Sa, and Nandini Singhal. A Simple and Practical Concurrent Non-blocking Unbounded Graph with Linearizable Reachability Queries. In Proceedings of the 20th International Conference on Distributed Computing and Networking, ICDCN 2019, Bangalore, India, January 04-07, 2019, pages 168-177, 2019.

[5] Thomas H Cormen, Charles E Leiserson, Ronald L Rivest, and Clifford Stein. Introduction to algorithms. MIT press, 2009.

[6] Nhan Nguyen Dang and Philippas Tsigas. Progress Guarantees when Composing Lock-free Objects. In Proceedings of the 17th International Conference on Parallel Processing - Volume Part II, Euro-Par'11, pages 148-159, 2011.

[7] Camil Demetrescu, Irene Finocchi, and Giuseppe F. Italiano. Dynamic graphs. In Handbook of Data Structures and Applications. 2004.

[8] Timothy L. Harris. A Pragmatic Implementation of Non-blocking Linked-Lists. In Distributed Computing, 15th International Conference, DISC 2001, Lisbon, Portugal, October 3-5, 2001, Proceedings, pages 300-314, 2001.

[9] Maurice Herlihy and Nir Shavit. On the Nature of Progress. In Principles of Distributed Systems - 15th International Conference, OPODIS 2011, Toulouse, France, December 13-16, 2011. Proceedings, pages 313-328, 2011.

[10] Maurice P. Herlihy and Jeannette M. Wing. Linearizability: a correctness condition for concurrent objects. ACM Trans. Program. Lang. Syst., 12(3):463-492, 1990.

[11] Nikolaos D. Kallimanis and Eleni Kanellou. Wait-Free Concurrent Graph Objects with Dynamic Traversals. In 19th International Conference on Principles of Distributed Systems, OPODIS 2015, December 14-17, 2015, Rennes, France, pages 27:1-27:17, 2015.

[12] Serguei Popov. The tangle, https://iota.org/iota whitepaper.pdf, 2018.

[13] Arnab Sinha and Sharad Malik. Runtime checking of serializability in software transactional memory. In 24th IEEE International Symposium on Parallel and Distributed Processing, IPDPS 2010, Atlanta, Georgia, USA, 19-23 April 2010 - Conference Proceedings, pages 1$12,2010$.

[14] Gerhard Weikum and Gottfried Vossen. Transactional Information Systems: Theory, Algorithms, and the Practice of Concurrency Control and Recovery. Morgan Kaufmann, 2002. 


\section{Appendix}

\section{A Proof of the Acyclicity}

Lemma 1 In any state, all the edges with the status ADDED are acyclic.

Proof Sketch: Suppose $G$ is a graph which is acyclic and all its edges are in the ADDED state before invocation of $\operatorname{ACY} \operatorname{ADDE}(k, l)$ by any thread $T_{x}$.

Let the $T_{i}$ and $T_{j}$ be the two threads concurrently invokes $\operatorname{Acy} \operatorname{AdDE}\left(k_{i}, l_{i}\right)$ and $\operatorname{AcY} \operatorname{AdDE}\left(k_{j}, l_{j}\right)$ respectively. Suppose both the edges are in the same directed path $v(l) \rightsquigarrow v(k)$ and both independently create cycle with their respective edge. We use $e\left(k_{i}, l_{i}, s t\right)$ to represent an edge, where $s t$ is the current status of the edge, it may be ADDED, TRANSIT or MARKED.

when $T_{i}$ invokes an $\operatorname{ACY} \operatorname{ADDE}\left(k_{i}, l_{i}\right)$ operation, first it checks the validations from the Line 5970. After successfully validations $T_{i}$ adds the edge $e\left(k_{i}, l_{i}\right.$, TRANSIT $)$ and then invokes the $\operatorname{SCRCBL}\left(l_{i}, k_{i}\right)$ to test cycle. Similarly, $T_{j}$ also added an $e\left(k_{j}, l_{j}\right.$, TRANSIT $)$ and invokes $\operatorname{SCRCBL}\left(l_{j}, k_{j}\right)$ to check a cycle. Both the threads return boolean status and the BFS-tree. The BFS-tree contains the edges which are not MARKED (means only the edges which are either ADDED or TRANSIT). Now, we have three cases:

- $e\left(k_{i}, l_{i}\right.$, TRANSIT $) \prec e\left(k_{j}, l_{j}\right.$, TRANSIT $)$ : Here $e\left(k_{i}, l_{i}\right.$, TRANSIT $)$ got added before $e\left(k_{j}, l_{j}\right.$, TRANSIT $)$. From our assumption that all the edges in the path $v(l) \rightsquigarrow v(k)$ are in the state ADDED and the graph $G$ is acyclic. Now $T_{i}$ added the edge $e\left(k_{i}, l_{i}\right.$, TRANSIT/ADDED) before $T_{j}$ adds the edge $e\left(k_{j}, l_{j}\right.$, TRANSIT) and then $T_{j}$ in the process of SCRCBL(in the Line 76 ). The $T_{j}$ collects the BFS-tree starting from $v\left(l_{j}\right)$ and it will consider the edge $e\left(k_{i}, l_{i}\right.$, TRANSIT/ADDED $)$. If it reaches $v\left(k_{j}\right)$ by using the path $v\left(l_{j}\right) \rightsquigarrow v\left(k_{j}\right)$ it simply discards its edge by setting the state from TRANSIT to MARKED in Line 81. Otherwise, it adds the edge by setting the state from TRANSIT to ADDED in Line 77. Hence this case cycle is not possible and the $G$ remain as acyclic.

- $e\left(k_{j}, l_{j}\right.$, TRANSIT $) \prec e\left(k_{i}, l_{i}\right.$, TRANSIT $)$ : Here $e\left(k_{j}, l_{j}\right.$, TRANSIT $)$ got added before $e\left(k_{i}, l_{i}\right.$, TRANSIT $)$. It is similar to the previous case, from our assumption that all the edges in the path $v(l)$ $\rightsquigarrow v(k)$ are in the state ADDED, all the vertices are reachable from the $v(l)$ and the graph $G$ is acyclic. Now $T_{j}$ added the edge $e\left(k_{j}, l_{j}\right.$, TRANSIT/ADDED) before $T_{i}$ adds the edge $e\left(k_{i}, l_{i}\right.$, TRANSIT) and then $T_{i}$ in the process of SCRCBL(in Line 76$)$ it will consider the edge $e\left(k_{j}, l_{j}\right.$, TRANSIT/ADDED). If it finds a path $v\left(l_{i}\right) \rightsquigarrow v\left(k_{i}\right)$ it simply discards its edge by setting the state from TRANSIT to MARKED in Line 81 . Hence this case cycle also not possible and the $G$ remain as acyclic.

- $e\left(k_{i}, l_{i}\right.$, TRANSIT $) \approx e\left(k_{j}, l_{j}\right.$, TRANSIT $):$ Here $e\left(k_{i}, l_{i}\right.$, TRANSIT $)$ got added approximately same time $e\left(k_{j}, l_{j}\right.$, TRANSIT $)$. From our assumption that all the edges in the path $v(l) \rightsquigarrow v(k)$ are in the state ADDED, all the vertices are reachable from the $v(l)$ and the graph $G$ is acyclic. Now $T_{i}$ and $T_{j}$ added the edge $e\left(k_{i}, l_{i}\right.$, TRANSIT $)$ and $e\left(k_{j}, l_{j}\right.$, TRANSIT $)$ at same time respectively. Then both $T_{i}$ and $T_{j}$ are in the process of SCRCBL(in Line 76). $T_{i}$ will consider the edge $e\left(k_{j}, l_{j}\right.$, TRANSIT) added by $T_{j}$ and it finds there is a path $v\left(l_{i}\right) \rightsquigarrow v\left(k_{i}\right)$ and then discards its edge by setting the state from TRANSIT to MARKED in Line 81. Similarly, $T_{j}$ will consider the edge $e\left(k_{i}, l_{i}\right.$, TRANSIT $)$ added by $T_{i}$ and it finds there is a path $v\left(l_{j}\right) \rightsquigarrow v\left(k_{j}\right)$ and then discards its edge by setting the state from TRANSIT to MARKED in the Line 81 . Hence this case cycle also not possible and the $G$ remain as acyclic. 


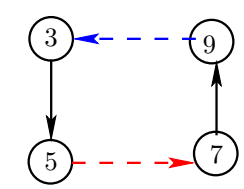

$T_{1}: \operatorname{AcyAddE}(5,7)$

$T_{2}: \operatorname{Acy} A d d \mathbf{E}(9,3)$

$T_{3}: \operatorname{AcyConE}(5,7)$

(a)

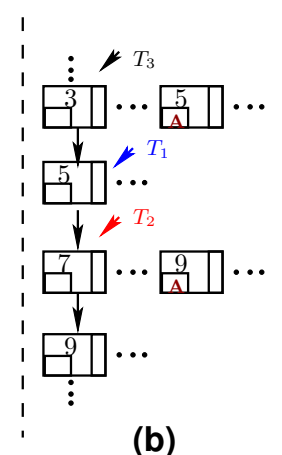

(b)

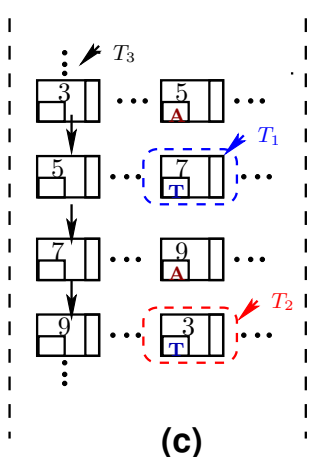

(c)

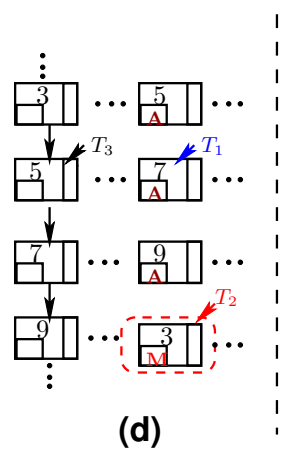

(d)

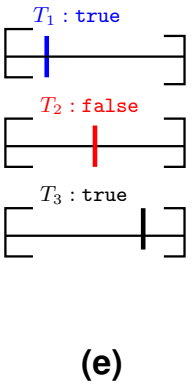

(e)

Figure 7: An example working of the methods while preserving acyclicity. (a) The initial graph, $T_{1}, T_{2}$ and $T_{3}$ are concurrently performing operations. The corresponding data-structure is shown in (b). In (c), $T_{3}$ is traversing the vertex list, while $T_{1}$ and $T_{2}$ have added their corresponding edges in TRANSIT, $T$ state and performing cycle detection. (d) $T_{1}$ has succeeded; and changed the status to ADDED, $A$. However, $T_{2}$ failed; it changes the status to MARKED, $M$. Meanwhile, $T_{3}$ finds the respective edge. (e) One possible linearization of this concurrent execution.

\section{B The Acyclic Graph Algorithms}




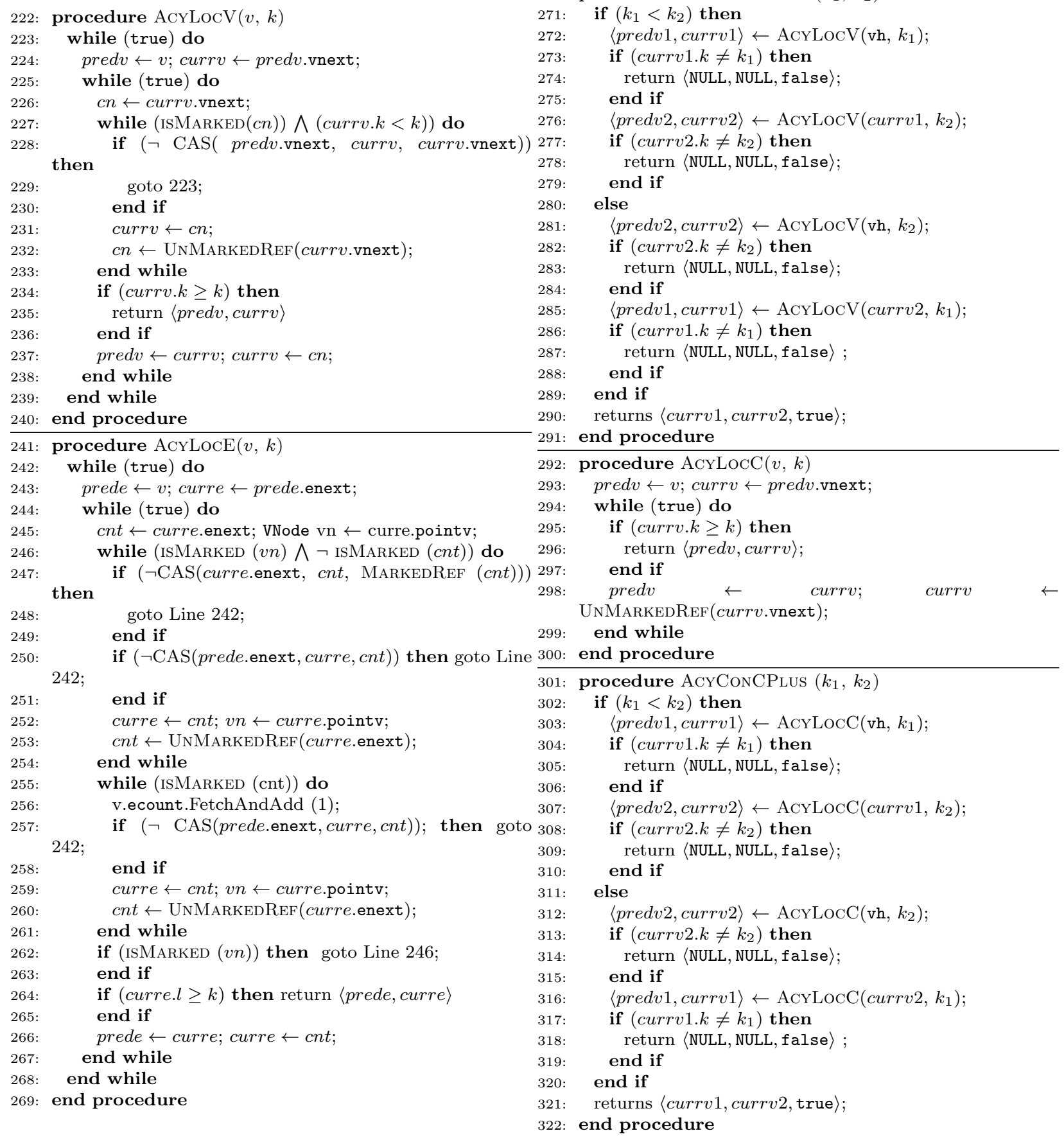

Figure 8: Pseudo-codes of AcyLocE, AcyConCPlus, AcylocC and AcyConCPlus. 